\title{
Primary Aldosteronism With Right-Dominant Heart Failure
}

\author{
Kazuro Sugishita, ${ }^{1}$ MD, Hironori Muraoka, ${ }^{1}$ MD, Akiko Kawana, ${ }^{1}$ MD, \\ Masako AsaKawa, ${ }^{1} \mathrm{MD}$, Shin-ichi Usur, ${ }^{1} \mathrm{MD}$, and Toshiyuki TAKaHASHI, ${ }^{1} \mathrm{MD}$
}

\begin{abstract}
SUMMARY
A 48 year-old obese male with hypertension was admitted to our department because of severe right-dominant heart failure. His heart rhythm was 2:1 atrial flutter and the left ventricle was diffusely hypertrophic and hypokinetic. Primary aldosteronism was diagnosed based on severe hypokalemia $(2.6 \mathrm{mEq} / \mathrm{L})$ and a low renin-high aldosterone state with hypertension despite the use of an angiotensin-II receptor blocker, but its etiology could not be clarified with computed tomography, adrenal scintigraphy, and adrenal vein sampling. Ascites and edema rapidly worsened. Ascites aspiration was performed daily, until serum potassium was normalized by a full dose of an aldosterone receptor blocker (spironolactone $100 \mathrm{mg} /$ day). A diuretic (furosemide) was then added. Rate control of atrial flutter was obtained with a beta-adrenergic blocker, and anticoagulation therapy was started. His heart failure was successfully controlled. Coronary arteries were normal on coronary arteriograms, and an endomyocardial biopsy sample obtained from the left ventricle did not show any specific pathological findings. Blood pressure was well controlled with the full dose of the aldosterone receptor blocker, but he died one year later due to intracerebral hemorrhage. As his heart failure was right dominant, we believe that its etiology may have been hyperaldosteronism-induced cardiomyopathy, and not advanced hypertensive heart disease. (Int Heart J 2010; 51: 293-297)
\end{abstract}

Key words: Cardiomyopathy, Hypokalemia, Ascites, Spironolactone

A ldosterone has recently been shown to play important roles, not only in the homeostasis of blood pressure and serum electrolyte levels, but also in several cardiovascular diseases, that is, inducing cardiac hypertrophy and fibrosis and vascular endothelial injury. ${ }^{1)}$ The importance of angiotensin-II, an upstream member of aldosterone in the renin-angiotensin-aldosterone system (RAAS), is already well recognized and its inhibition in patients with cardiovascular diseases is widely performed in clinical practice. Aldosterone production, however, has been reported to be incompletely blocked with angiotensin-II inhibition only (aldosterone breakthrough). ${ }^{2,3)}$ Two clinical studies have reported the beneficial effects of aldosterone receptor antagonist use combined with standard therapy consisting of angiotensin-II blockade in patients with heart failure. ${ }^{4,5}$

Patients with primary aldosteronism, because of its high plasma aldosterone levels, are considered to be at high risk of developing cardiovascular diseases. We present here a case with primary aldosteronism with right-dominant heart failure. This case provides interesting information with respect to assessing the pathophysiological roles of aldosterone in cardiovascular diseases.

\section{Case Report}

A 48 year-old male was referred to our department due to worsened leg edema and abdominal distension that had persisted for a couple of months and a few weeks, respectively.
He was obese and had mild diabetes mellitus, hyperuricemia, and sleep apnea syndrome. He had been taking an angiotensinII receptor blocker (telmisartan $40 \mathrm{mg} /$ day) for several years to treat his hypertension. He did not smoke, but his parents were also hypertensive. Chest X-rays showed an enlarged cardiac silhouette, and electrocardiograms (ECG) demonstrated atrial flutter with 2:1 atrioventricular conduction. An aldosterone receptor blocker (spironolactone $25 \mathrm{mg}$ /day) was started for his severe hypokalemia. He was admitted to our department the next day.

At admission, his body weight was $123.7 \mathrm{~kg}$ (body mass index, 39.9), which was an increase of approximately $25 \mathrm{~kg}$ from his baseline body weight (body mass index, 31.6). His blood pressure and pulse rate were $176 / 122 \mathrm{mmHg}$ and 120 beats per minutes (bpm), respectively. Exophthalmos was suspected, and his skin was wet. Neither heart murmurs nor respiratory rales were audible. Abdominal distension was remarkable, but there was neither spontaneous pain nor tenderness. Pretibial pitting edema was also severe in both legs. Laboratory data (Table) showed mild renal dysfunction (serum creatinine concentration $1.5 \mathrm{mg} / \mathrm{dL}$, creatinine clearance $62 \mathrm{~mL} /$ minute). Severe hypokalemia (serum potassium concentration $2.8 \mathrm{mEq} /$ L) was found despite the administration of both angiotensin-II and aldosterone receptor blockers. Serum uric acid was very high $(12.0 \mathrm{mg} / \mathrm{dL})$, and his diabetes mellitus was poorly controlled (HbAlc 7.6\%). We suspected hyperthyroidism because of the exophthalmos, poorly controlled diabetes, and hypocholesterolemia, however, his thyroid function was almost within normal limits. His plasma brain natriuretic peptide (BNP) level

From the ${ }^{1}$ Department of Cardiology, JR Tokyo General Hospital, Tokyo, Japan.

Address for correspondence: Kazuro Sugishita, MD, Department of Cardiology, JR Tokyo General Hospital, 2-1-3 Yoyogi, Shibuya-ku, Tokyo 151-8528, Japan.

Received for publication April 30, 2010.

Revised and accepted May 20, 2010. 
Table.

\begin{tabular}{|c|c|c|c|c|c|c|}
\hline WBC & 7000/uL & $\mathrm{Na}$ & $147 \mathrm{mEq} / \mathrm{L}$ & $\mathrm{H}$ & $<$ Urine $>$ & \\
\hline $\mathrm{Neu}$ & $76.1 \%$ & K & $2.8 \mathrm{mEq} / \mathrm{L}$ & $\mathrm{L}$ & $\mathrm{pH}$ & 6.5 \\
\hline Lym & $14.9 \%$ & $\mathrm{Cl}$ & $97 \mathrm{mEq} / \mathrm{L}$ & & Pro & $(2+) \rightarrow(-)$ \\
\hline Mon & $4.7 \%$ & T-Chol & $111 \mathrm{mg} / \mathrm{dL}$ & $\mathrm{L}$ & Glu & $(-)$ \\
\hline Eos & $1.7 \%$ & TG & 93 mg/dL & & Ket & $(-)$ \\
\hline Baso & $0.9 \%$ & HDL & $32 \mathrm{mg} / \mathrm{dL}$ & $\mathrm{L}$ & Bil & $(-)$ \\
\hline $\mathrm{RBC}$ & $538 \times 10^{4} / \mathrm{uL}$ & LDL & $63 \mathrm{mg} / \mathrm{dL}$ & $\mathrm{L}$ & $\mathrm{OB}$ & $(-)$ \\
\hline $\mathrm{Hb}$ & $15.2 \mathrm{~g} / \mathrm{dL}$ & Glu & $107 \mathrm{mg} / \mathrm{dL}$ & & Uro & $\mathrm{N}$ \\
\hline Het & $45.7 \%$ & AST & $33 \mathrm{IU} / \mathrm{L}$ & & & \\
\hline Plt & $30 \times 10^{4} / \mathrm{uL}$ & ALT & $23 \mathrm{IU} / \mathrm{L}$ & & $\mathrm{CCr}$ & $62 \mathrm{~mL} / \mathrm{min} \mathrm{L}$ \\
\hline PT & $70 \% \quad \mathrm{~L}$ & LD & $240 \mathrm{IU} / \mathrm{L}$ & $\mathrm{H}$ & & \\
\hline PT-INR & 1.2 & $\gamma$ GTP & $41 \mathrm{IU} / \mathrm{L}$ & & & \\
\hline APTT & $34 \mathrm{sec}$ & CK & 237 IU/L & & & \\
\hline $\mathrm{TP}$ & $7.1 \mathrm{~g} / \mathrm{dL}$ & CRP & $0.68 \mathrm{mg} / \mathrm{dL}$ & $\mathrm{H}$ & TSH & $1.35 \mathrm{uU} / \mathrm{mL}$ \\
\hline Alb & $4.0 \mathrm{~g} / \mathrm{dL}$ & $\mathrm{HbAlc}$ & $7.6 \%$ & $\mathrm{H}$ & fT3 & $2.42 \mathrm{pg} / \mathrm{mL}$ \\
\hline BUN & 16.3 mg/dL & & & & fT4 & $1.63 \mathrm{ug} / \mathrm{dL} \quad \mathrm{H}$ \\
\hline UA & $12.0 \mathrm{mg} / \mathrm{dL} \mathrm{H}$ & BNP & $180 \mathrm{pg} / \mathrm{mL}$ & $\mathrm{H}$ & & \\
\hline Cre & $1.5 \mathrm{mg} / \mathrm{dL} \mathrm{H}$ & & & & & \\
\hline
\end{tabular}

Laboratory data at admission demonstrated mild renal dysfunction and severe hypokalemia. Diabetes mellitus was poorly controlled, and serum uric acid was very high. Thyroid function was almost within normal limits. Plasma brain natriuretic peptide levels were mildly elevated at admission, and increased further to $628 \mathrm{pg} / \mathrm{mL}$ during hospitalization. Proteinuria observed at admission subsequently improved. WBC indicates white blood cell, Neu; neutrophil, Lym; lymphocyte, Mon; monocyte, Eos; eosinophil, Baso; basophil, RBC; red blood cell, $\mathrm{Hb}$; hemoglobin, Hct; hematocrit, Plt; platelet, PT; prothrombin time, PT-INR; prothrombin time international normalized ratio, APTT; activated partial thromboplastin time, TP; total protein, Alb; albumin, BUN; blood urea nitrogen, UA; uric acid, Cre; creatinine, Na; sodium, K; potassium, Cl; chloride, T-Chol; total cholesterol, TG; triglycerides, HDL; high density lipoprotein, LDL; low density lipoprotein, Glu; glucose, AST; aspartate aminotransferase, ALT; alanine aminotransferase, LD; lactate dehydrogenase, $\gamma$ GTP; gamma-glutamyl transpeptidase, CK; creatine phosphokinase, CRP; C-reactive protein, HbAlc; hemoglobin Alc, BNP; brain natriuretic peptide, Pro; protein, Ket; ketone, Bil; bilirubin, $\mathrm{OB}$; occult blood, Uro; urobilinogen, $\mathrm{CCr}$; creatinine clearance, TSH; thyroid stimulating hormone, fT3; free triiodothyronine, and fT4; free thyronine.

A

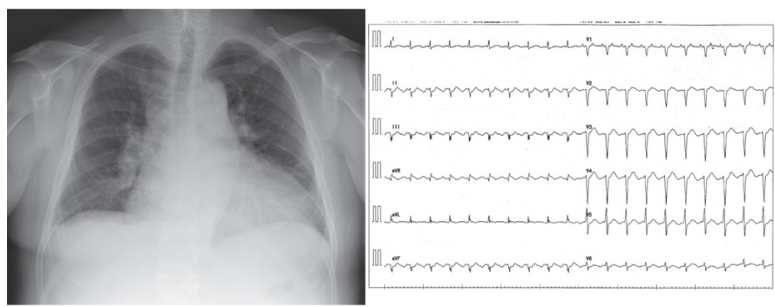

Figure 1. Chest x-ray and electrocardiogram (ECG) obtained at admission. Chest x-ray (A) demonstrated enlargement of cardiac silhouette with pulmonary artery dilatation or hilar lymph node swelling, and ECG (B) showed 2: 1 atrial flutter with left axis deviation and poor $\mathrm{R}$ wave progression in the anterior chest leads.

was mildly elevated $(180 \mathrm{pg} / \mathrm{mL})$ at admission, and increased further to $628 \mathrm{pg} / \mathrm{mL}$ during hospitalization. Proteinuria observed at admission subsequently improved. Chest $\mathrm{x}$-rays demonstrated an enlarged cardiac silhouette with pulmonary artery dilatation or hilar lymph node swelling (Figure 1A), and ECG showed a 2:1 atrial flutter with left axis deviation and poor $\mathrm{R}$ wave progression in the anterior chest leads (Figure 1B). Ultrasonocardiograms (UCG) revealed severe left ventricular (LV) hypertrophy (interventricular septal thickness 18
A

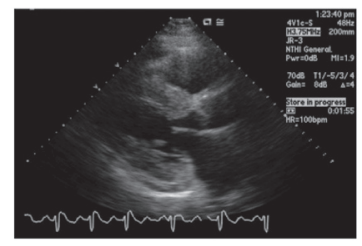

C

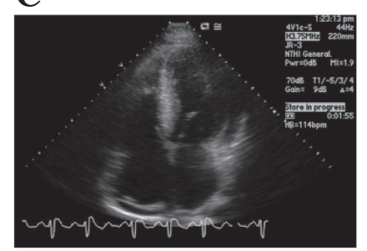

B

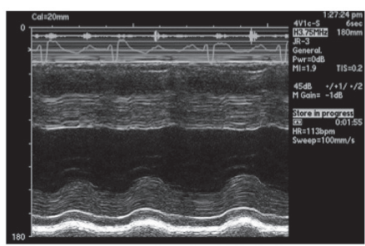

D

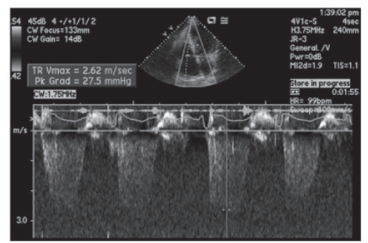

Figure 2. Ultrasonocardiogram (UCG) at admission. Long axis view (A), m-mode (B), four chamber view (C), and tricuspid regurgitation pattern (D). Left ventricle (LV) showed severe hypertrophy (interventricular septal thickness $18 \mathrm{~mm}$, posterior wall thickness $19 \mathrm{~mm}$ ) and diffuse hypokinesis with dilatation (diastolic $\mathrm{LV}$ diameter $58 \mathrm{~mm}$, LV ejection fraction 45\%) (A, B). Left atrium (LA) was severely dilated (LA diameter $70 \mathrm{~mm}$ ) (A, C). Right ventricular systolic pressure was estimated to be high (47 $\mathrm{mmHg})(\mathbf{D})$ 


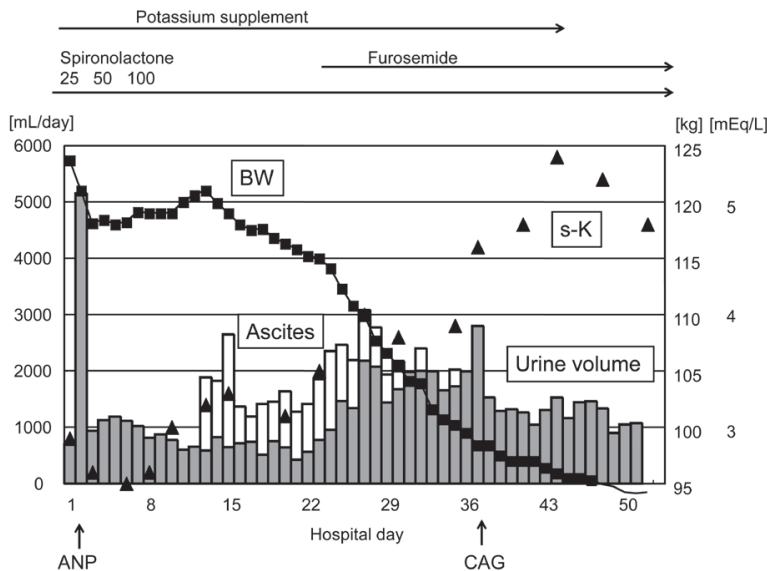

Figure 3. In-hospital clinical course. At admission, intravenous atrial natriuretic peptide (carperitide $0.025 \mu \mathrm{g} / \mathrm{kg} / \mathrm{min}$ ) administration was started, but discontinued the next day because of an excessive diuretic reaction and worsening of hypokalemia. The dose of spironolactone was gradually increased to $100 \mathrm{mg}$ a day and potassium was added. However, the ascites and edema deteriorated rapidly so the ascites (approximately $1 \mathrm{~L} /$ day) was drained almost daily beginning on hospital day 12. Furosemide was started on hospital day 22 when the serum potassium level was increased to 3.5 $\mathrm{mEq} / \mathrm{L}$, and an adequate diuretic reaction was obtained. Daily ascites drainage was terminated on hospital day 34 (total of $17 \mathrm{~L}$ for 25 days). Potassium supplementation was ceased on hospital day 44 and the hypokalemia did not recur. BW indicates body weight, s-K; serum potassium concentration, ANP; atrial natriuretic peptide; and CAG; coronary arteriogram.
A

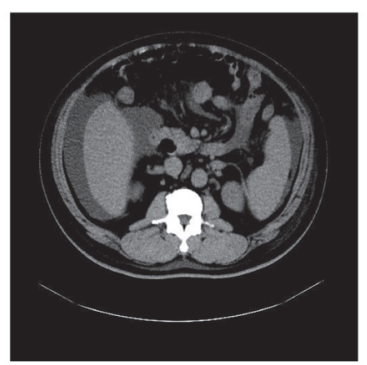

B

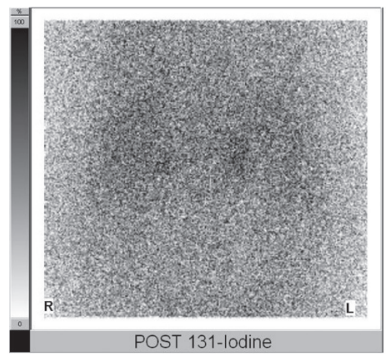

Figure 4. Abdominal computed tomogram (CT) (A) and adrenal scintigram (B). Neither of these tests detected any abnormal findings on his bilateral adrenal glands.

$\mathrm{mm}$, posterior wall thickness $19 \mathrm{~mm}$ ) and diffuse LV wall hypokinesis with LV dilatation (diastolic LV diameter $58 \mathrm{~mm}$, LV ejection fraction 45\%) (Figure 2A, 2B). The left atrium (LA) was also severely dilated (LA diameter $70 \mathrm{~mm}$ ) (Figure 2A, 2C). Right ventricular systolic pressure was estimated to be high $(47 \mathrm{mmHg}$ ) (Figure 2D). We started intravenous atrial natriuretic peptide (carperitide $0.025 \mu \mathrm{g} / \mathrm{kg} /$ minute) administration to treat his severe overhydration and hypertension, however, it was discontinued the next day because of an excessive diuretic reaction (urine volume $>5 \mathrm{~L} /$ day) and worsening of the hypokalemia $(2.6 \mathrm{mEq} / \mathrm{L})$ (Figure 3$)$. Administration of the beta-adrenergic blocker bisoprolol ( $2.5 \mathrm{mg} /$ day) was started to control the rate of atrial flutter due to concern that digitalis
A

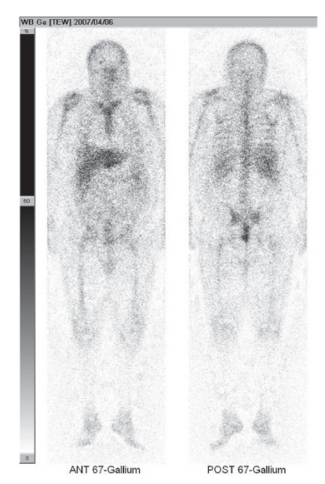

B

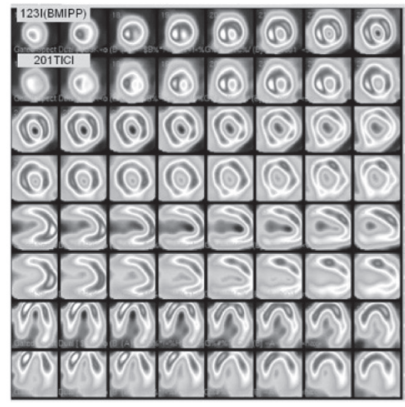

Figure 5. Gallium scintigram (A) and myocardial dual scintigram (B). Neither of these tests demonstrated any abnormal findings. BMIPP indicates beta-methyl-p-iodophenyl-petadecanic acid and $\mathrm{TlCl}$; Thallium chloride.

toxicity might be induced in his hypokalemic state. The dose of spironolactone was gradually increased up to $100 \mathrm{mg} /$ day, with potassium supplementation as appropriate, to treat his severe hypokalemia. However, the ascites and edema, but not lung congestion, rapidly worsened so we had to drain the ascites (approximatedly 1L/day) almost daily beginning on the $12^{\text {th }}$ day of hospitalization (day 12). After the serum potassium level was normalized to $3.5 \mathrm{mEq} / \mathrm{L}$ on day 22 , intravenous furosemide administration was started, resulting in an adequate diuretic reaction. The ascites and edema gradually improved. The final ascites drainage was performed on day 34 (a total of $17 \mathrm{~L}$ was removed in 25 days). On that day, his body weight was already decreased by $25 \mathrm{~kg}$ compared with that on admission and the serum potassium level was increased to $3.9 \mathrm{mEq} /$ L (Figure 3). As his severe hypertension and hypokalemia remained despite the angiotensin-II receptor and aldosterone blockage, we suspected primary aldosteronism. In fact, his blood sample demonstrated extremely low renin and high aldosterone; plasma renin activity (PRA) was $0.4 \mathrm{ng} / \mathrm{mL} / \mathrm{hour}$, plasma aldosterone concentration (PAC) was $447 \mathrm{pg} / \mathrm{mL}$, and the PAC/PRA ratio was 1117.5. We therefore diagnosed him as having primary aldosteronism, but neither computed tomography (CT) (Figure 4A) nor adrenal scintigraphy (Figure 4B) detected any abnormal findings on his bilateral adrenal glands. Concerning the etiology of his heart disease, we suspected infiltrative heart disease, the dilated phase of hypertrophic cardiomyopathy, or end stage hypertensive heart disease, because of his diffusely hypertrophic and hypokinetic LV. Although cardiac sarcoidosis and heart-originated malignant lymphoma were suspected because of mild mediastinal lymph node swelling on chest CT (not shown), his serum angiotensin converting enzyme level was normal $(12.4 \mathrm{u} / \mathrm{L})$ and serum soluble interleukin 2 receptor level was only mildly elevated $(717 \mathrm{u} / \mathrm{mL})$. A gallium scintigram (Figure 5A) and myocardial dual scintigram (Figure 5B) did not demonstrate any abnormal findings. A cardiac catheter test was performed on hospital day 36 . A hemodynamic study showed normal cardiac output (cardiac index $2.6 \mathrm{~L} / \mathrm{minute} / \mathrm{m}$ ), but elevated pulmonary arterial wedge pressure $(23 \mathrm{mmHg}), \mathrm{LV}$ end-diastolic pressure $(25 \mathrm{mmHg})$, and right ventricular end-diastolic pressure $(15 \mathrm{mmHg})$, de- 

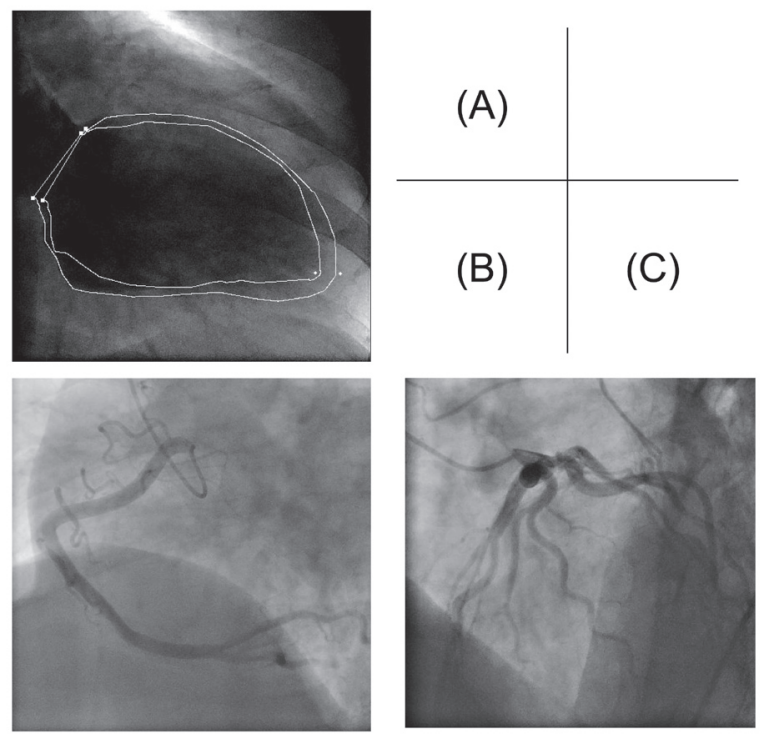

Figure 6. Cardiac catheter test on the 36th hospital day. Left ventriculogram demonstrated severe diffuse LV wall hypokinesis with severe low ejection fraction (26\%) (A). Coronary arteriogram (right; B, left; C) revealed normal coronary arteries.

spite marked $(25 \mathrm{~kg})$ body weight loss with diuretics. A left ventriculogram demonstrated severe diffuse LV wall hypokinesis with a very low ejection fraction (26\%) (Figure 6A). Coronary arteriograms revealed normal coronary arteries (Figure 6B, 6C). Left ventricular endomyocardial biopsy was performed, but a pathological examination did not detect any specific findings in the samples (not shown). A transesophageal ultrasonocardiogram showed the possible presence of atrial thrombosis so warfarin administration was started. Since his serum potassium level was increased after the catheter test, potassium supplementation was discontinued on hospital day 44 . The hypokalemia did not recur. He declined to undergo electrical defibrillation and catheter ablation to treat the atrial flutter, and was discharged on hospital day 54.

Aldosterone receptor blocker (spironolactone $100 \mathrm{mg}$ / day), beta-adrenergic blocker (bisoprolol $2.5 \mathrm{mg} /$ day), angiotensin-II receptor blocker (telmisartan $40 \mathrm{mg} /$ day), diuretic (furosemide $20 \mathrm{mg} /$ day), and warfarin administration were continued. His blood pressure at the outpatient clinic was stable at approximately $120 / 80 \mathrm{mmHg}$, and his international normalized ratio of prothrombin time (PT-INR) was controlled at 1.5-2.0. Decompensated heart failure did not reappear (Figure 7). One year later, the atrial flutter had changed to atrial fibrillation, but UCG showed no remarkable improvement of LV systolic function (diastolic LV diameter $56 \mathrm{~mm}$, LV ejection fraction 49\%). Three months later, he experienced sudden right hemiplegia and aphasia, and was rushed to another hospital. A brain CT revealed an intracerebral hemorrhage (not shown). The size of the hematoma had increased rapidly due to the warfarin therapy. Emergency neurosurgery was performed, but he died 3 days later. An autopsy was not performed.

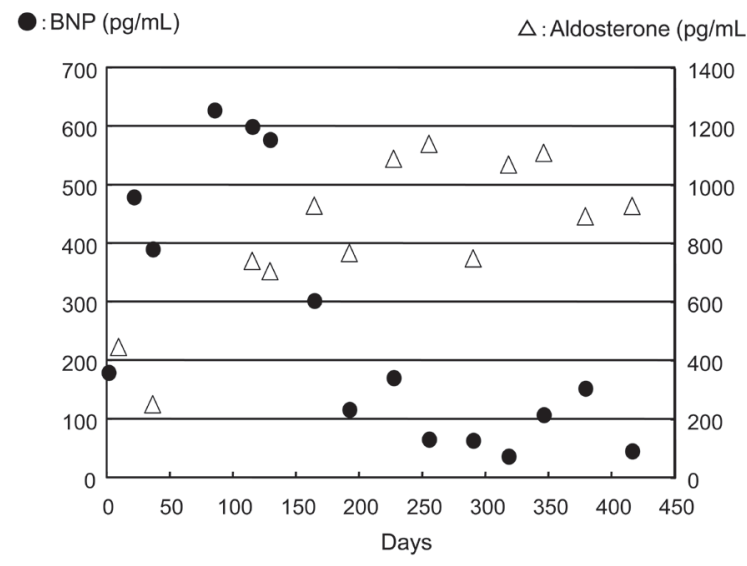

Figure 7. Time courses of plasma brain natriuretic peptide (BNP) and aldosterone levels. BNP levels did not increase again for one year after discharge, although the high aldosterone levels continued.

\section{Discussion}

The renin-angiotensin-aldosterone system (RAAS) plays important roles, not only in the homeostasis of blood pressure and serum electrolytes, but also in several cardiovascular diseases. Angiotensin-induced cardiovascular injury has been well elucidated, and the beneficial effects of its inhibition with angiotensin-converting enzyme (ACE) inhibitors or angiotensin-II receptor blockers (ARB) have been well documented in experimental and clinical studies. However, recently it has been reported that aldosterone production was not completely blocked by inhibition of angiotensin-II, which is upstream of aldosterone in the RAAS (aldosterone breakthrough). ${ }^{2,3)}$ Aldosterone was reported to induce cardiac hypertrophy and fibrosis as well as vascular endothelial injury in experimental studies. ${ }^{1)}$ Clinical studies, specifically the Randomized Aldosterone Evaluation Study (RALES) ${ }^{4}$ and Eplerenone Neurohormonal Efficacy and Survival Study (EPHESUS), ${ }^{5}$ also reported that the addition of aldosterone receptor antagonists to the standard therapy with ACE inhibitors significantly improved the prognosis of patients with heart failure. Based on these reports, patients with primary aldosteronism are considered to be at high risk of developing a cardiovascular disease, and to be interesting with respect to assessing the pathophysiological roles of aldosterone in cardiovascular diseases.

The diagnosis of primary aldosteronism in the present case was considered to be definitive because of the hypertension accompanied by severe hypokalemia (minimum serum potassium concentration $2.5 \mathrm{mEq} / \mathrm{L}$ ) and extraordinarily high plasma aldosterone concentration/plasma renin activity (PAC/ PRA) ratio (1117.5), although these data were obtained during angiotensin-II receptor blocker (telmisartan) and aldosterone receptor blocker (spironolactone) administration. We could not identify the etiology of the primary aldosteronism in this patient even though we performed abdominal CT, adrenal scintigraphy, and adrenal venous sampling. Recently, being overweight or obese has been shown to be associated with increased aldosterone levels. ${ }^{6}$ Adipose tissue or fatty acids may stimulate the production of aldosterone. As the present case was obese (baseline body mass index of 31.6) before the onset of heart 
failure and after the compensation of heart failure, his hyperaldosteronism might have been related to his fat tissue.

Atrial fibrillation was reported to be present much more frequently in patients with primary aldosteronism than in those with essential hypertension. Watson, et al proposed 3 possible mechanisms for this association: left ventricular hypertrophy due to hypertension, hypokalemia, and cardiac fibrosis due to aldosterone excess. ${ }^{8)}$ To the best of our knowledge, atrial flutter has never been reported to be related to primary aldosteronism, and this paper is the first case report describing an association between these two diseases. We suspect that atrial flutter may also be induced by the above 3 possible mechanisms.

The left ventricle of the present case showed typical diffuse hypertrophy and diffuse hypokinesis in clinical tests that included UCG and LVG. However, the endomyocardial biopsy samples from the left ventricle did not show any pathological findings typical of hypertensive heart disease or aldosteroneinduced myocardial disease, that is, myocyte hypertrophy or intercellular fibrosis. As the sensitivity of endomyocardial biopsy through a catheter is known to be not high, this cardiac disorder was diagnosed as advanced hypertensive heart disease due to primary aldosteronism or hyperaldosteronism-induced cardiomyopathy. In addition, the heart failure of the present case was right-dominant with severe ascites and edema. $\mathrm{He}$ showed only mild lung congestion. His ascites and edema worsened after the initiation of a beta-adrenergic blocker for rate control of atrial flutter after admission, but his dyspnea and lung congestion did not worsen during this period. Since hyperaldosteronism may induce potent volume expansion, but not edema, the severe ascites and edema in the present case were considered to be signs of right heart failure. Although there was also pulmonary hypertension probably secondary to left ventricular dysfunction at admission, right ventricular systolic pressure estimated with UCG at admission was not high enough $(47 \mathrm{mmHg})$ to induce severe right heart failure. Hemodynamic study revealed that both LV end-diastolic pressure $(25 \mathrm{mmHg})$ and right ventricular end-diastolic pressure were elevated $(15 \mathrm{mmHg})$. Hypertensive heart disease is generally considered to induce left ventricular hypertrophy against systemic hypertension and subsequent left ventricular diastolic dysfunction, and finally to cause left ventricular systolic dysfunction. On the other hand, aldosterone may affect the biventricular myocardium. Therefore, we concluded the etiology of his heart failure was hyperaldosteronism-induced biventricular cardiomyopathy.

Atrial natriuretic peptide (ANP) has been reported to show higher plasma concentrations in patients with primary aldosteronism, ${ }^{9,10)}$ and its infusion was revealed to induce more potent natriuretic effects in these cases. ${ }^{11,12)}$ Rocco, et al reported that ANP administration increased not only urinary volume and urinary sodium excretion, but also urinary potassium excretion during the first hour in patients with primary aldosteronism. ${ }^{12)}$ The forced cessation of intravenous ANP administration in the present case because of excessive diuretic effects and worsened hypokalemia was compatible with the results of earlier reports.
Intracerebral hemorrhage resulted in his sudden death. Since an autopsy was not performed and we did not obtain detailed information about the emergency neurosurgery from the other hospital, we cannot rule out a congenital intracerebral vascular disease, such as an aneurysm or arteriovenous malformation. However, long-term secondary hypertension due to primary aldosteronism and direct vascular injury by aldosterone $^{1)}$ may have been related to his intracerebral hemorrhage.

Finally, the present patient with right-dominant heart failure is interesting in terms of the pathophysiological roles of aldosterone in cardiovascular diseases, because, to our knowledge, this is the first report of right-dominant heart failure due to hyperaldosteronism. Expression of aldosterone receptors (mineralocorticoid receptors) might have been augmented in the right ventricle of this patient, although we are unable to discuss this possibility any further because we are not in possession of any right ventricular myocardial samples.

\section{REFERENCES}

1. Yoshimoto T, Hirata Y. Aldosterone as a cardiovascular risk hormone. Endocr J 2007; 54: 359-70. (Review)

2. Struthers AD. Aldosterone escape during angiotensin-converting enzyme inhibitor therapy in chronic heart failure. J Card Fail 1996; 2: 47-54. (Review)

3. McKelvie RS, Yusuf S, Pericak D, et al. Comparison of candesartan, enalapril, and their combination in congestive heart failure: randomized evaluation of strategies for left ventricular dysfunction (RESOLVD) pilot study. The RESOLVED Pilot Study Investigators. Circulation 1999; 100: 1056-64.

4. Pitt B, Zannad F, Remme WJ, et al. The effect of spironolactone on morbidity or mortality in patients with severe heart failure. Randomized Aldactone Evaluation Study Investigators. N Engl J Med 1999; 341: 709-17

5. Pitt B, Remme W, Zannad F, et al. Eplerenone, a selective aldosterone blocker, in patients with left ventricular dysfunction after myocardial infarction. N Engl J Med 2003; 348: 1309-21.

6. Vogt B, Bochud M, Burnier M. The association of aldosterone with obesity-related hypertension and the metabolic syndrome. Semin Nephrol 2007; 27: 529-37. (Review)

7. Milliez P, Girerd X, Plouin PF, Blacher J, Safar ME, Mourad JJ. Evidence for an increased rate of cardiovascular events in patients with primary aldosteronism. J Am Coll Cardiol 2005; 45: 1243-8.

8. Watson T, Karthikeyan VJ, Lip GY, Beevers DG. Atrial fibrillation in primary aldosteronism. J Renin Angiotensin Aldosterone Syst 2009; 10: 190-4.

9. Yamaji T, Ishibashi M, Sekihara H, Takaku F, Nakaoka H, Fujii J. Plasma levels of atrial natriuretic peptide in primary aldosteronism and essential hypertension. J Clin Endocrinol Metab 1986; 63: 815-8.

10. Hirata Y, Ishii M, Sugimoto T, et al. Hormonal and renal effects of atrial natriuretic peptide in patients with secondary hypertension. Circulation 1988; 78: 1401-10.

11. Mantero F, Rocco S, Pertile F, Carpené G, Fallo F, Menegus A. Alpha-h-ANP injection in normals, low renin hypertension and primary aldosteronism. J Steroid Biochem 1987; 27: 935-40.

12. Rocco S, Opocher G, Carpenè G, Mantero F. Atrial natriuretic peptide infusion in primary aldosteronism. Renal, hemodynamic and hormonal effects. Am J Hypertens 1990; 3: 668-73. 